\title{
Rapid Quantification of Fungicide Effectiveness on Inhibiting Wheat Stripe Rust Pathogen (Puccinia striiformis f. sp. tritici)
}

\author{
Furong Peng, Miu Si, Yang Zizhu, Yuhang Fu, Yuheng Yang, Yang Yu, and Chaowei Bi ${ }^{\dagger}$ \\ College of Plant Protection, Southwest University, Chongqing 400715, P.R. China
}

\begin{abstract}
Wheat stripe rust, caused by Puccinia striiformis f. sp. tritici (Pst), is one of the most important and devastating diseases of wheat; therefore, it is necessary to rapidly and accurately quantify fungicide effectiveness to monitor Pst sensitivity and manage the disease. In this study, a rapid method of quantifying the fungicide effectiveness with detached leaves was developed. The results showed that $0.5 \%$ water agar containing $75 \mu \mathrm{g} / \mathrm{ml}$ of 6-benzylaminopurine and filter paper worked the best for maintaining wheat leaves. The disease incidences of different concentrations of spore suspension were compared. When the spore

in the efficacies of triadimefon on rust suppression between detached leaves in the culture dishes and direct spray on seedlings. We also developed a Photoshop software method that can replace the current classification method and accurately measure the proportion of sporulation area on infected leaves. The sensitivity baseline of Pst to triadimefon was estimated as $0.1453 \pm 0.0081 \mu \mathrm{g} / \mathrm{ml}$, and all the values of $\mathrm{EC}_{50}$ were tested for normal distribution using the Shapiro-Wilk test ( $W=$ $0.204)$. The baseline can be used to test the sensitivity of different $P s t$ isolates to triadimefon.
\end{abstract} concentrations were 5 and $10 \mathrm{mg} / \mathrm{ml}$, the disease incidences had no significant differences at 12 and 15 days after inoculation $(P<0.05)$. Fungicide treatment tests revealed that there were no significant differences
Keywords: Puccinia striiformis f. sp. tritici, triadimefon, detached leaves, fungicide effectiveness quantification
The obligate biotrophic fungus Puccinia striiformis Westend. f. sp. tritici Erikss. (Pst) is an economically important pathogen that causes wheat stripe rust. Yield losses in wheat from Pst infections are usually the result of reduced kernel number per spike, lowered weight, and reduced grain quality (He et al. 2019). Pst is one of the most serious biotic threats to global wheat production (Wellings 2011). Stripe rust is especially a serious problem in China (Chen et al. 2014). Chemical control and growing resistant cultivars are the main methods used for controlling wheat stripe rust (Chen 2014), but integrating fungicides, resistance, and cultivation measures is a widely accepted strategy (Yang et al. 2017).

According to the China Pesticide Information Network, the fungicides for controlling wheat rusts mainly include triazoles belonging to the demethylation inhibitor (DMI) group, carboxamides in the succinate dehydrogenase inhibitor group, and methoxy acrylate mitochondrial inhibitors. DMIs are the most widely used fungicides in the world, and they account for the largest proportion of fungicides used in controlling wheat rusts. Triadimefon was the first DMI fungicide marketed by Bayer in 1976. The number of products containing triadimefon is still larger than the other triazole agents in China. Triadimefon has the characteristics of high efficiency, low toxicity, broad spectrum, systemic absorption, and long-lasting effect. It entered field testing in 1972 and was used for control of powdery mildew in 1976 (Siegel 1981). Triadimefon has a strong therapeutic effect on wheat stripe rust and can also be used to treat wheat powdery mildew and leaf rust (Lin 1982). This fungicide can interrupt

${ }^{\dagger}$ Corresponding author: C. W. Bi; chwbi@swu.edu.cn

Funding: This work was supported by the National Key R\&D Program of China (2018YFD0200500), National Natural Science Foundation of China (31871990, 31801719), and Fundamental Research Funds for the Central Universities (XDJK2017B026).

The author(s) declare no conflict of interest.

Accepted for publication 14 March 2020.

(C) 2020 The American Phytopathological Society the infection by killing mycelia of the pathogens in wheat leaves during the incubation period (Shang and Lu 1991).

Many research institutes and enterprises have been studying, innovating, and screening new fungicides that are effective in controlling wheat stripe rust. An effectiveness assay is a prerequisite for fungicide screening and fungicide resistance assessment. Most effectiveness measurements of fungicides are carried out via the growth of pathogens in vitro, and monitoring needs to be carried out strictly to prevent development of fungicide resistance and prolong the service life of the fungicides (Grimmer et al. 2014). However, as an obligate parasite, Pst is presently not possible to be cultured in vitro. Thus, the traditional mycelial growth method cannot be used to measure the actual effectiveness of fungicides on this pathogen (CSICNY 2006a).

The potted plantlets test (CSIC-NY 2006b), seed dressing, and detached leaf segment methods have been used to assess the sensitivity to fungicides of the powdery mildew pathogens, which are similar to Pst in their obligate specificity of parasitisms. The sensitivity of assaying various rust fungi, such as coffee leaf rust (Hemileia vastatrix) (Belan et al. 2015), beet rust (Uromyces beticola), and wheat leaf rust (Puccinia triticina), was measured using tests on field plants. The fungicidal effectiveness was studied by estimating the differences in the lesion areas (CSIC-NY 2008) or the number of infected sites between treated and untreated control plants. However, these methods used for evaluating pathogen sensitivity to fungicides are subjected to the environment. Inoculation by seed dressing is usually started when a wheat plantlet completely unfolds at the first leaf, but this requires the fungicide being effective for a rather long duration. The efficacy of fungicides is also considerably affected by other factors including soils and moisture (Khandelwal et al. 2014; Wang et al. 2017). The data measured vary remarkably for individuals, and the trial is labor-intensive and requires repetitive work to adjust the accuracy of data.

A method for determining the effectiveness of fungicides against wheat powdery mildew on detached leaves has been described by the Fungicide Resistance Action Committee (FRAC) (https:// www.frac.info/). First, potted young plants were sprayed with fungicide, and the leaf segments were cut and placed in a fresh medium for spore suspension inoculation. The disease suppressive effect was measured by counting the infected sites. The sensitivity of cucumber downy mildew to fungicide was determined by inoculating the 
detached leaf segments suspended on the fungicide-containing liquid with the spore suspension (Liu 2004). The detached leaf bioassay of fungicides against obligate parasites is convenient, repeatable, and involving much less workload than potted plant tests and traditional field trials. However, our preliminary experiments suggested that these methods cannot be directly applied to measure the effectiveness of fungicides against wheat stripe rust because of changes in ambient humidity that affect urediniospore germination and hyphal penetration of stomata.

Although a method for assessing fungicide effectiveness of Pst (the potted plant test) was published by FRAC and China Pesticide Indoor Bioassay Test Guidelines (CSIC-NY NY/T 1156.15-2008; CSIC-NY 2008), a guideline for determining and comparing the effectiveness of different fungicides against wheat stripe rust in vitro with less plant materials and labor cost needs to be established to provide a theoretical basis for screening new fungicides and for monitoring field development of fungicide-resistant strains more conveniently and efficiently.

\section{Materials and Methods}

Rust fungal inoculum. The original isolates of Pst were collected from field wheat plants in Hechuan District of Chongqing (N30 $10^{\prime}$ $\left.24^{\prime \prime}, \mathrm{E} 106^{\circ} 25^{\prime} 37^{\prime \prime}\right)$ in 2018 . At the initial period of stripe rust development, wheat leaves with single or few sparsely distributed uredinia were collected. The samples were stored at $4^{\circ} \mathrm{C}$ in the laboratory, and fresh urediniospores were collected from uredinia on these leaf samples immediately before inoculation.

The collected leaves were rinsed with running water to wash off the spores from the uredinia on the surface and were placed in Petri dishes with moist filter paper. The Petri dishes were placed in a growth chamber $\left(12^{\circ} \mathrm{C}\right.$ and $16 \mathrm{~h} /$ day of light at 11,000 luxes with luminous bars) until sporulation. Urediniospores of $P s t$ were isolated using a sterile needle and inoculated by shaking off the urediniospores from the needle onto the seedling, and each seedling was inoculated with spores from only one uredinium. The inoculated plants were maintained in the dark and saturated by enclosing them in black plastic bags for $24 \mathrm{~h}$ after inoculation and grew for 15 days in a greenhouse before urediniospores were collected. The urediniospores harvested from each seedling were considered as an individual isolate. Each isolate was propagated and multiplied by inoculating wheat seedlings with its urediniospores in a greenhouse. The newly produced spores were harvested in a centrifuge tube and stored at $4^{\circ} \mathrm{C}$ for later use.

Chemicals. Gibberellic acid (GAs), 6-benzylaminopurine (6-BA) (Bai et al. 2018), and benzimidazole (BEN) were tested as the freshmaintaining agents for keeping detached wheat leaves for bioassay uses. Triadimefon is a fungicide commercially applied for controlling stripe rust in the field. Related details of these chemicals are presented in Table 1.

Wheat plants. Wheat (cv. Mingxian 169) seeds were soaked in water overnight and germinated on wet blotting paper at room temperature in darkness. Germinated seeds were sown in the mixture of peat soil and vermiculite in plastic pots $(7 \mathrm{~cm}$ on a side). The pots were placed in a greenhouse $\left(17^{\circ} \mathrm{C}, 16 \mathrm{~h} /\right.$ day of light at 11,000 luxes $)$. Ten uniform healthy young seedlings were retained in each pot, and the others were rooted out at the first leaf stage.

Screening chemical agent for maintaining wheat leaves. Wheat leaves were maintained in vitro to meet the growth condition of Pst. Three chemical agents were added separately with different concentrations to sterilized water, and $20 \mathrm{ml}$ of each mixture was put into a 9-cm Petri dish. The wheat leaves were cut into approximately 1.0$\mathrm{cm}$ segments and suspended in the dish. The concentrations of the agents were set as follows: GA at 10,20 , and $30 \mu \mathrm{g} / \mathrm{ml} ; 6-\mathrm{BA}$ at $25,50,75$, and $100 \mu \mathrm{g} / \mathrm{ml}$; and BEN at 50, 65, 75, and $100 \mu \mathrm{g} / \mathrm{ml}$. Each treatment was repeated three times. The green color preservation time and complete yellowing time were recorded.

Screening fresh-maintaining substrate. Using the best freshmaintaining agent and its concentration obtained from the previous experiment, different cushions were compared. These included water, two-layer filter paper, $0.5 \%$ water agar, $1 \%$ water agar, and $0.5 \%$ water agar combined with filter paper. Each treatment was repeated three times, and the green color preservation and complete yellowing time were recorded.

Selecting fresh-maintaining medium for urediniospore germination. Sterile water, $0.5 \%$ water agar, and $0.5 \%$ water agar with $75 \mu \mathrm{g} / \mathrm{ml}$ of 6 -BA ( $250 \mu \mathrm{l}$ in each case) were separately added to concave glass slides. Fresh urediniospores and talc powder $(\mathrm{m} 1 /$ $\mathrm{m} 2=1: 3$ ) were mixed in a test tube, and the urediniospore mixture was shaken evenly onto the slides, which were placed in a porcelain dish for moisturizing. After 3 and $6 \mathrm{~h}$ of dark incubation at $17^{\circ} \mathrm{C}$, the slides were observed under a microscope for checking spore germination. Ten views $(200 \times)$ were observed for each slide. The number of germinated urediniospores (germ tube length $>$ short diameter of the spore) was recorded, and germination rate (\%) was calculated. The test was repeated four times.

Screening urediniospore concentration for inoculating Pst on wheat seedlings. Urediniospores were weighed and mixed with $10 \mathrm{ml}$ of $0.02 \%$ Tween 20 with a vortex shaker for 5 min to prepare spore suspension. The inoculum suspension was placed in an airbrush (HD-130), and $5 \mathrm{ml}$ of suspension was used to inoculate three pots. The tested concentrations of suspension were $00.5,1,5$, and $10 \mathrm{mg} / \mathrm{ml}$, and the control group was treated by spaying $5 \mathrm{ml}$ of $0.02 \%$ Tween 20 solution. The incubation period (the period from the establishment of parasitic relationship after the pathogen invades the host to the occurrence of obvious symptoms) and rust incidence were recorded at 12 and 15 days after inoculation. To provide an estimate of urediniospores sprayed on the seedlings, three glass coverslips were placed near the seedlings simultaneously. After inoculation, the coverslips were placed under a microscope $(100 \times)$ to count the number of urediniospores. Five fields were observed for each slide. The test was repeated four times.

Selecting the method of fungicide treatment on detached leaf segments. The detached leaves infected by Pst were treated with triadimefon ( 1 and $5 \mu \mathrm{g} / \mathrm{ml}$ ) in three ways: (i) spraying detached leaves using an atomizer, (ii) immersing detached leaves in the fungicide solution for $2 \mathrm{~h}$, and (iii) placing leaf segments directly on the fresh-maintaining medium containing triadimefon. The seedlings were sprayed directly with the fungicide as the control. All the treated plants were maintained for $12 \mathrm{~h}$ at saturation humidity and darkness and then grown in a growth chamber. When the infected leaf area reached $80 \%$ on control plants, the proportion of sporulating area for all treatments was evaluated using Photoshop.

Fungicide efficacy test. Urediniospores of each isolate were mixed with $10 \mathrm{ml}$ of $0.02 \%$ Tween 20 in a $15-\mathrm{ml}$ centrifuge tube, and five pots of wheat seedlings were inoculated by spraying the urediniospore mixture with an airbrush (HD-130). The inoculated plants were kept for $24 \mathrm{~h}$ at $100 \%$ wetness in darkness and then grown in a $17^{\circ} \mathrm{C}$ greenhouse until being used for the fungicide efficacy test below.

Table 1. Chemicals used in this study

\begin{tabular}{lcccc}
\hline Active ingredients & CAS & Formulation $^{\mathbf{z}}$ & Manufacturer & Abbreviation \\
\hline Triadimefon & $43121-43-3$ & $95 \% \mathrm{TC}$ & KangBaoTai, Hubei & $\ldots$ \\
Gibberellic acid & $77-06-5$ & $90+\% \mathrm{AR}$ & Angene, Nanjing & GA \\
6-Benzylaminopurine & $1214-39-7$ & $98+\%$ AR & Bide Pharmatech, Shanghai & 6-BA \\
Benzimidazole & $51-17-2$ & $98.5 \% \mathrm{AR}$ & Macklin, Shanghai & BEN \\
\hline
\end{tabular}

${ }^{\mathrm{z}} \mathrm{TC}=$ technical material, and $\mathrm{AR}=$ analytical reagent. 
To prepare the fungicide-containing fresh-maintaining media, $75 \mu \mathrm{g} / \mathrm{ml}$ of $6-\mathrm{BA}$ and different concentrations (0.03, 0.09, $0.27,0.81,2.43,7.29$, and $21.87 \mu \mathrm{g} / \mathrm{ml}$ ) of triadimefon were added to $0.5 \%$ water agar. No fungicide was added in the freshmaintaining medium as the control. Then, $20 \mathrm{ml}$ of the medium

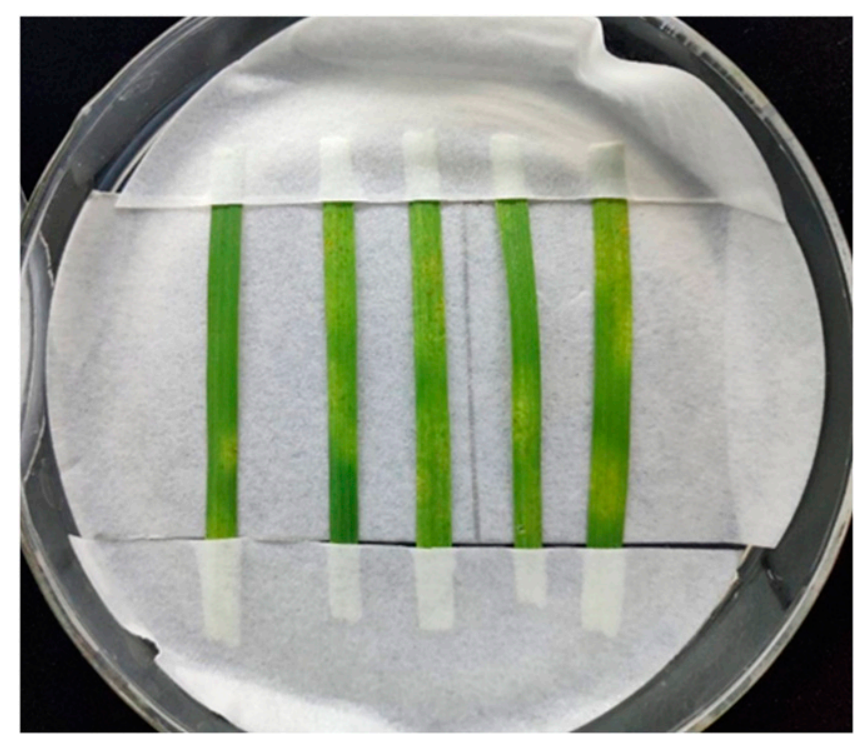

Fig. 1. The treatment of wheat leaf segments. was added into a Petri dish, and a filter paper (about $8 \times 4 \mathrm{~cm}$ ) was placed on the surface of the solid medium. Leaf segments of $5 \mathrm{~cm}$ in length from rusted seedlings were prepared, and five segments were situated and fixed on the filter paper in each medium plate (Fig. 1). The dishes were incubated in a $12^{\circ} \mathrm{C}$ growth chamber (with $16 \mathrm{~h}$ of light per day), and the proportion of sporulating area on the leaf segments was assessed and recorded 8 days after treatment using Photoshop (Fig. 2). The test was repeated three times.

Data analysis. The virulence regression equation $Y=a X+b$, where $X$ is the logarithm of fungicide concentration and $Y$ is the probability value of fungal growth inhibition, was established using Excel software. A frequency histogram (median effective concentration [EC $\left.{ }_{50}\right]$ of the strains was the $X$ axis, and the distribution frequency was the $Y$ axis) was obtained, and thus the trend line on the histogram was the sensitivity baseline (Felsenstein et al. 1998). The obtained values were subjected to normal Shapiro-Wilk $(W)$ test using the SPSS software package.

\section{Results}

Screening chemical agent for maintaining wheat leaves. Among the three chemicals (BEN, 6-BA, and GA) tested, 6-BA at a concentration of $75 \mu \mathrm{g} / \mathrm{ml}$ had the best result of maintaining detached wheat leaves fresh, and the green color of the leaves was retained for 19 days after treatment. This was followed by BEN with a green color-retention time of 16 days at $100 \mu \mathrm{g} / \mathrm{ml}$, and GA resulted in the shortest retention time, 15 days at $30 \mu \mathrm{g} / \mathrm{ml}$ (Fig. 3). Given that Pst infections can develop in wheat leaves until the leaves become completely yellow, $75 \mu \mathrm{g} / \mathrm{ml}$ of 6-BA was a significant improvement

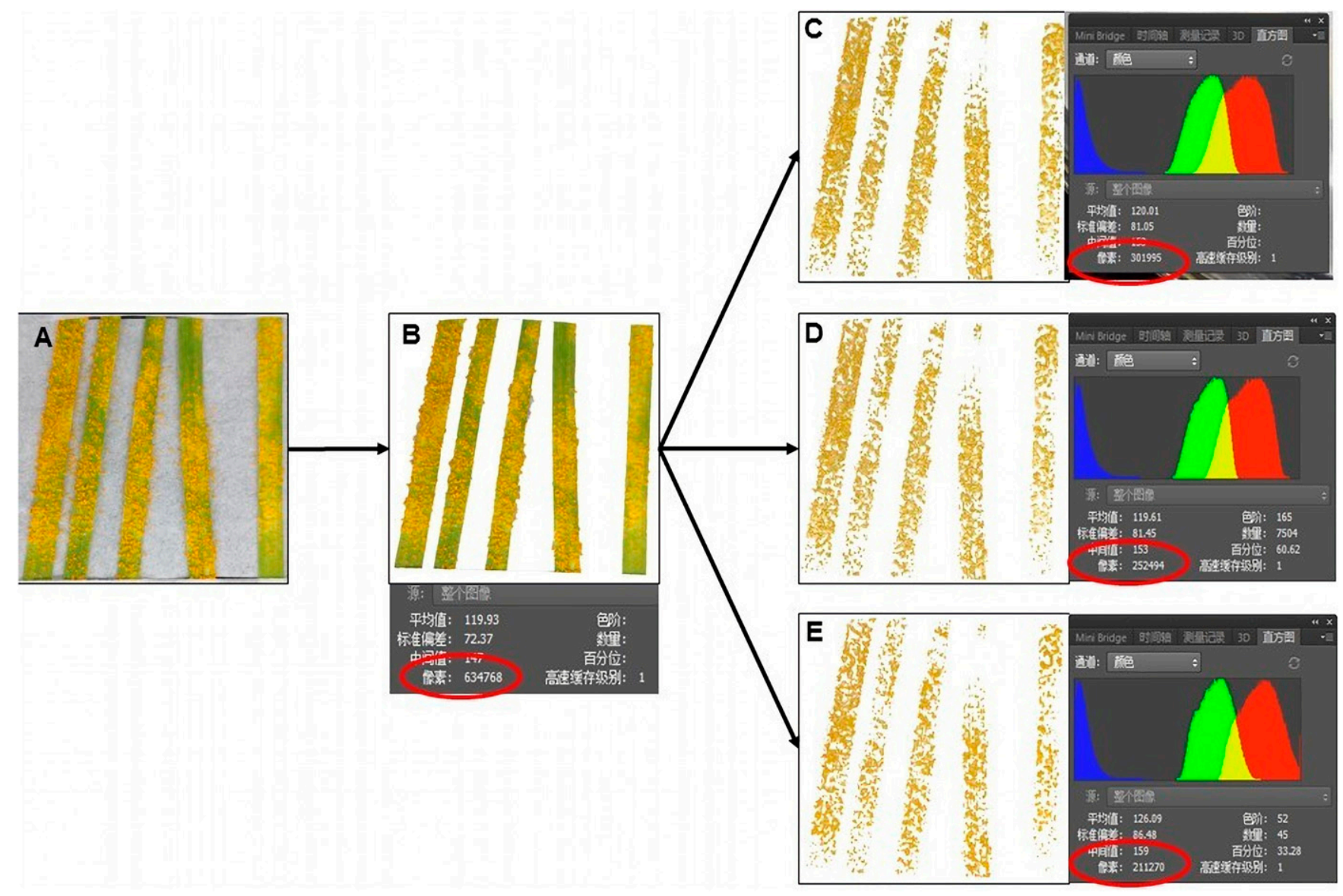

Fig. 2. Schematic diagram of measuring the proportion of infected sites by Photoshop software. A, Picture of the blank control group (isolates of no. 39) taken with a Canon camera; B, the total pixels of five leaf segments are 634,768 ; C, the pixels of urediniospores are 301,995 with a tolerance of 70 ; D, the pixels of urediniospores are 252,494 with a tolerance of $60 ; \mathrm{E}$, the pixels of urediniospores are 211,270 with a tolerance of 50 . (Tolerance is the selection range set when the color is selected: the larger the tolerance, the larger the selected range.) The proportion of infected sites $=[(301,995+252,494+211,270) / 3] / 634,768=40.21 \%$. 
over BEN and GA. So, $75 \mu \mathrm{g} / \mathrm{ml}$ 6-BA was used in all subsequent detached leaf treatments.

Screening fresh-maintaining substrate. When the different substrates were compared using $75 \mu \mathrm{g} / \mathrm{ml}$ of 6 -BA as the greening agent, the combined substrate of $0.5 \%$ water agar and filter paper had the best preservation effect of detached wheat leaves (Fig. 4), and the detached leaves stared yellowing in 12 days and completely yellowed in 19 days following treatment. The green effect with water agar was also satisfactory, and the color of the leaf segment was completely yellowed in 18 days. The detached leaves in the double-layer filter paper treatment were completely yellowed after 16 days, and those in the $1 \%$ water agar treatment were completely yellowed after 15 days. Therefore, the combination of $0.5 \%$ water agar mixed with $75 \mu \mathrm{g} / \mathrm{ml}$ of $6-$ BA and filter paper was used to preserve the detached leaves in the further tests.

Selecting fresh-maintaining medium for urediniospore germination. Compared with using sterile water as the control, both $0.5 \%$ water agar and $75 \mu \mathrm{g} / \mathrm{ml}$ of 6-BA had no significant effect on Pst urediniospore germination (Table 2). The spores germinated normally, and the germination rates in all treatments reached more than $80 \%$ after $6 \mathrm{~h}$.

Screened urediniospore concentration for inoculating Pst on wheat seedlings. Urediniospore productions between repeatedly observed slides of a spore concentration treatment were roughly the same when the spore suspension was sprayed with an airbrush, and this implicated that the spore suspension was evenly distributed on wheat seedlings sprayed. However, productions between different spore concentrations $(0.5,1.0,5.0$, and $10 \mathrm{mg} / \mathrm{ml})$ were extremely varied, and the spore production increased as the inoculation concentration was raised (Table 3 ).

When the stripe rust incidences and the disease incubation periods were recorded on wheat seedlings 12 and 15 days after inoculation and compared between spore concentrations, the inoculation at 0 and $0.5 \mathrm{mg} / \mathrm{ml}$ concentrations produced no rust symptom or sign, but the higher concentrations $(1.0,5.0$, and $10.0 \mathrm{mg} / \mathrm{ml})$ resulted in different rust incidences and incubation periods on the inoculated seedlings. It was clear that higher inoculum density induced greater production of urediniospores and shorter disease incubation period, but the disease incidences between the concentrations of 5 and $10 \mu \mathrm{g} / \mathrm{ml}$ were not significantly different 15 days after inoculation (Table 4). Thus, the inoculum density $10 \mu \mathrm{g} / \mathrm{ml}$ spore suspension was chosen for the inoculation test.

Selected method of fungicide treatment on detached leaf segments. Three methods for applying triadimefon (by spraying, soaking leaf segments, and mixing in the medium) were tested in vitro, and the fungicidal effectiveness on suppressing stripe rust on detached wheat leaf segments was observed (Fig. 5). The infected leaf-area of the potted seedlings was more than $90 \%$ of the total leaf area after 13 days of inoculation, and it was $80 \%$ after 18 days of 8 days in vitro, in the no fungicide control treatment. At the concentration of $1 \mu \mathrm{g} / \mathrm{ml}$ of triadimefon, the three methods of fungicide application had no significant difference in rust disease suppression effect. At the concentration of $5 \mu \mathrm{g} / \mathrm{ml}$ of triadimefon, there was no significant difference of disease suppressions between the spraying of potted wheat seedlings and culturing inoculated leaf segments in vitro on medium containing the fungicide, whereas there was a significant difference in rust suppression between spraying potted seedlings and soaking leaf segments in the fungicide suspension. Therefore, the fungicide-containing medium culture method was used to treat the detached leaves in the later experiment for quantifying rust suppression effect.

Quantifying rust suppression effect. The reactions of 81 Pst isolates to triadimefon were tested in the experiment using the optimized technique. The $\mathrm{EC}_{50}$ of these isolates to the fungicide ranged from 0.0015 to $0.3117 \mu \mathrm{g} / \mathrm{ml}$ with an average of $0.1453 \pm 0.0081 \mu \mathrm{g} /$ $\mathrm{ml}$. The frequency histogram (group spacing of $0.02 \mu \mathrm{g} / \mathrm{ml}$ ) (Fig. 6) shows that the frequency distribution of the sensitivity of the 81 isolates to triadimefon is a continuous curve with a single peak. The frequency distribution was approximately normal $(W=0.204)$.
Therefore, the measured $0.1453 \pm 0.0081 \mu \mathrm{g} / \mathrm{ml}$ could be treated as a baseline for testing the sensitivity of Pst to triadimefon in the detached assays. This method could be used to measure the effectiveness of the fungicide against $P s t$ in vitro.

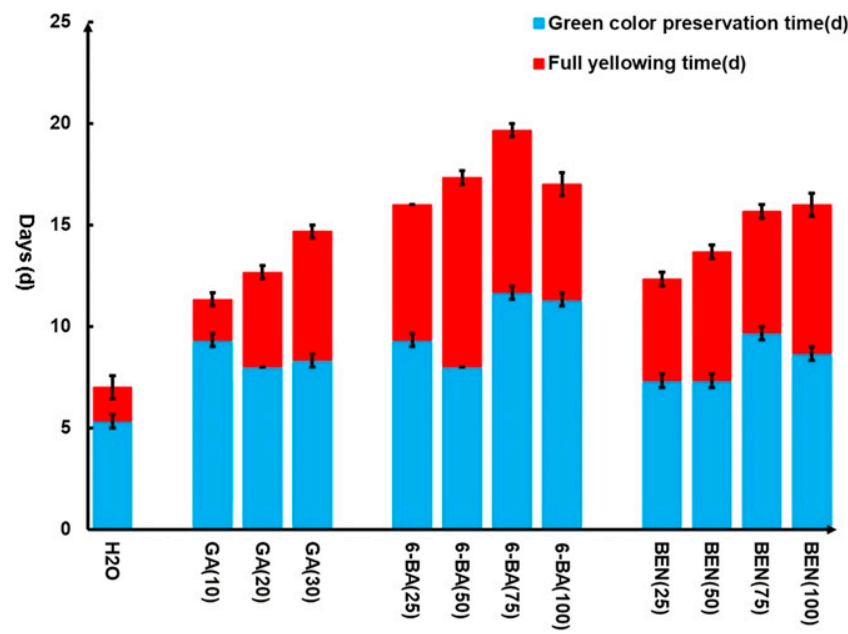

Fig. 3. Fresh-maintaining time of 1-cm detached wheat leaf segments under various concentrations of preservatives. $\mathrm{GA}=$ gibberellic acid; $\mathrm{BEN}=$ benzimidazole; and 6 $\mathrm{BA}=6$-benzylaminopurine. The values in parentheses are the concentrations of preservatives $(\mu \mathrm{g} / \mathrm{ml})$.

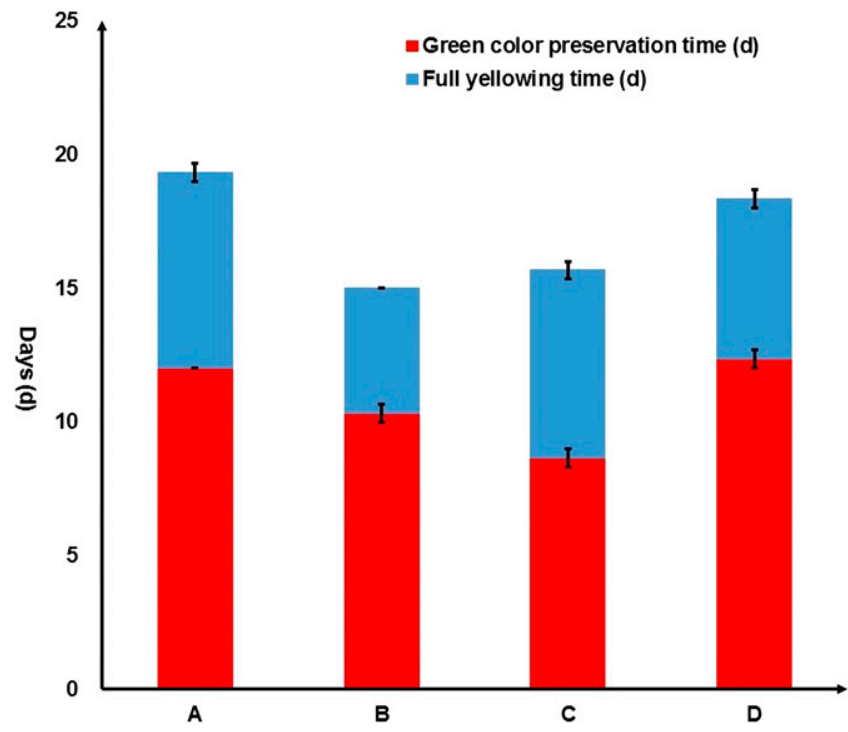

Fig. 4. Fresh-maintaining time in $75 \mu \mathrm{g} / \mathrm{ml}$ of $6-B A$ in different substrates of $5-\mathrm{cm}$ leaf segments of wheat in vitro. Five-centimeter detached leaves were situated on substrates: $A=0.5 \%$ water agar plus a layer of filter paper; $B=1 \%$ water agar plus a layer of filter paper; $\mathrm{C}=$ double-layer of filter paper; and $\mathrm{D}=$ water agar.

Table 2. Effect of preservatives on the germination of urediniospores of $P u c$ cinia striiformis f. sp. tritici

Urediniospore germination rate $(\%)^{\mathrm{y}}$

\begin{tabular}{lcc}
\cline { 2 - 3 } Treatments & $\mathbf{3 ~ h}$ & $\mathbf{6 ~ h}$ \\
\hline Water & $34.33 \pm 3.38 \mathrm{a}$ & $86.00 \pm 1.73 \mathrm{~b}$ \\
$0.5 \%$ water agar & $37.67 \pm 0.88 \mathrm{a}$ & $86.33 \pm 2.40 \mathrm{~b}$ \\
$0.5 \%$ water agar $+6-\mathrm{BA}(75)^{\mathrm{z}}$ & $31.00 \pm 3.21 \mathrm{a}$ & $82.67 \pm 1.45 \mathrm{~b}$ \\
\hline
\end{tabular}

${ }^{y}$ Different letters indicate significant difference at $P<0.05$ level by Duncan's new multiple range test.

z 6-BA (75) = 6-benzylaminopurine at $75 \mu \mathrm{g} / \mathrm{ml}$. 


\section{Discussion}

Although various studies have focused on elucidating the effectiveness of fungicides against wheat stripe rust by potting and field trials, more rapid, reliable, reproducible, and inexpensive determination of effectiveness provides an immediate benefit to agricultural stakeholders. A previous study evaluated the in vitro leaf assay for small germplasm screening of leaf rust resistance, and the leaf rust fungus had similar infection types on detached leaves and seedling plants (Boydom et al. 2013). Therefore, an in vitro leaf test was used to assess the genotype of wheat leaf rust resistance by Sergiev et al. (2007). Differences between the infection types of a detached leaf assay and intact plant assay were not significant and were mainly due to the senescence of the detached leaves, thereby confirming the effectiveness of the detached leaf assay (Patial et al. 2017).

The purpose of the present study was to find a method for measuring the sensitivity of $P$ st to fungicide using detached wheat leaves. The issues here were mainly concerned with the fresh preservation of the leaf segments, the fungal inoculation, fungicide treatment of detached leaves, and combining the various conditions to achieve as reliable results as those of pot-grown plant tests. Initially, a leaf disc suspension method was used, but because a long incubation of Pst was necessary and the detached leaves would be completely immersed in water after about 5 days, this method was not conducive to subsequent fungicide effect tests. In the current experiment, the $0.5 \%$ water agar $+6-\mathrm{BA}+$ filter paper was selected and used to preserve the leaf segments. This method was verified as easy to operate, and subsequent data measurements were made within a limited time period.

For inoculation of detached leaves, methods such as artificial rubbing, brushing (spore powder or suspension), spraying, dusting, and quantitative inoculation by means of a mechanical inoculation or sedimentation tower were tried, but the results showed that the leaf segments were rarely infected or that little disease symptom could be observed under general growing conditions. In these tests, leaves inoculated at different times were stained with trypan blue, and microscopic observations revealed that although the spores germinated and formed germ tubes, infection failed to occur. This result might be due to the changes in humidity or to metabolic and physiological conditions that resulted in stomatal closures on the detached leaves. In

Table 3. Number of urediniospores inoculated using an airbrush under an optical microscope $(200 \times)$

\begin{tabular}{|c|c|c|c|c|}
\hline \multirow[b]{2}{*}{$\begin{array}{l}\text { Spore suspension } \\
\text { concentration }(\mathrm{mg} / \mathrm{ml})\end{array}$} & \multicolumn{3}{|c|}{$\begin{array}{c}\text { Number of } \\
\text { urediniospores }\end{array}$} & \multirow[b]{2}{*}{$\begin{array}{c}\text { Mean number of } \\
\text { spores }^{\mathrm{z}}\end{array}$} \\
\hline & $\begin{array}{c}\text { Slide } \\
1\end{array}$ & $\begin{array}{c}\text { Slide } \\
2 \\
\end{array}$ & $\begin{array}{c}\text { Slide } \\
\mathbf{3}\end{array}$ & \\
\hline 0.5 & 1.60 & 1.00 & 1.80 & $1.47 \pm 0.24 \mathrm{a}$ \\
\hline 1.0 & 5.00 & 4.60 & 6.33 & $5.31 \pm 0.52 b$ \\
\hline 5.0 & 27.80 & 26.40 & 18.67 & $24.29 \pm 2.84 \mathrm{c}$ \\
\hline 10.0 & 48.40 & 39.33 & 43.80 & $43.84 \pm 2.62 \mathrm{~d}$ \\
\hline
\end{tabular}

${ }^{z}$ Different letters indicate significant difference at $P<0.05$ level by Duncan's new multiple range test.

Table 4. Disease incubation period and disease incidence after spray inoculation of different concentrations of urediniospore suspension

\begin{tabular}{lccc}
\hline Spore suspension & & \multicolumn{2}{c}{ Disease incidence $^{\mathbf{z}}$} \\
\cline { 3 - 4 } $\begin{array}{l}\text { concentration } \\
(\mathbf{m g} / \mathbf{m l})\end{array}$ & $\begin{array}{c}\text { Disease incubation } \\
\text { period (days) }\end{array}$ & $\mathbf{1 2}$ days & $\mathbf{1 5}$ days \\
\hline 0 & Not tested & 0.00 & 0.00 \\
0.5 & Not tested & 0.00 & 0.00 \\
1.0 & 10 & $15.95 \pm 1.62 \mathrm{a}$ & $42.22 \pm 3.98 \mathrm{a}$ \\
5.0 & 8 & $51.74 \pm 4.37 \mathrm{~b}$ & $79.05 \pm 6.62 \mathrm{~b}$ \\
10.0 & 7 & $63.75 \pm 5.91 \mathrm{~b}$ & $91.33 \pm 4.67 \mathrm{~b}$ \\
\hline
\end{tabular}

z Different letters indicate significant difference at $P<0.05$ level by Duncan's new multiple range test. addition, these changes were thought to affect fungicide movement in detached sugarcane leaves and spore germination (Chaulagain et al. 2019). Therefore, it was decided in the present study that wheat leaves were detached after $P s t$ inoculation and the spore density was optimized. It was hypothesized that the tolerance to the fungicide would increase when larger amounts of urediniospore inoculum were used, and as expected, better efficacy was observed when smaller amounts of inoculum were used (Pfaller et al. 2004). The amount of starting inoculum was considered as the single most important variable. After that, inoculation of 5 to $10 \mu \mathrm{g} / \mathrm{ml}$ of spore suspension by an HD-130 airbrush with an atomizer could distribute uniformly and thus ensured consistent infected area on leaf segments in a given culture period.

Fungicide was added to the medium as in the mycelial growth rate method. Although the incubation period of disease on the detached leaves was longer than that on intact pot-grown seedling plants, it was found that the rust control effects of 1 and $5 \mu \mathrm{g} / \mathrm{ml}$ of triadimefon on Pst in detached leaf assays were concordant with the results obtained from the traditional pot experiment. A previous paper

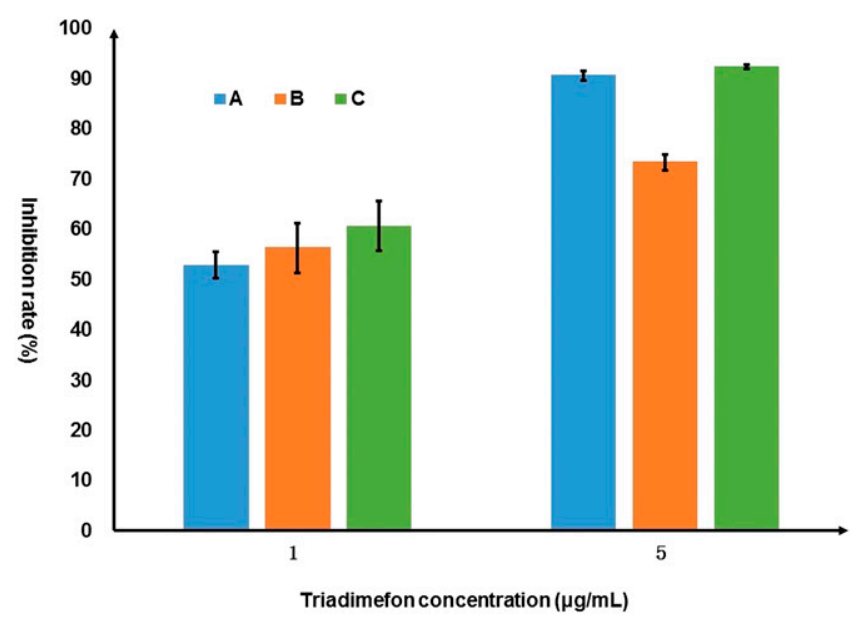

Fig. 5. Control effect of 1 and $5 \mu \mathrm{g} / \mathrm{ml}$ of triadimefon on wheat stripe rust in different treatments. $A=$ Pot-grown seedlings sprayed with 1 and $5 \mu \mathrm{g} / \mathrm{ml}$ of triadimefon; data of seedlings after 13 days of inoculation ( 8 days after fungicide treatment) were recorded. B = Soaking wheat leaf segments with 1 and $5 \mu \mathrm{g} / \mathrm{ml}$ of triadimefon for $2 \mathrm{~h}$; the leaves were placed in a fresh-maintaining plate, and data were recorded 18 days after inoculation ( 8 days after use of fungicide). $C=$ Leaf segments placed on fresh-maintaining medium containing 1 and $5 \mu \mathrm{g} / \mathrm{ml}$ of triadimefon; data were recorded 18 days after inoculation (8 days after fungicide spraying).

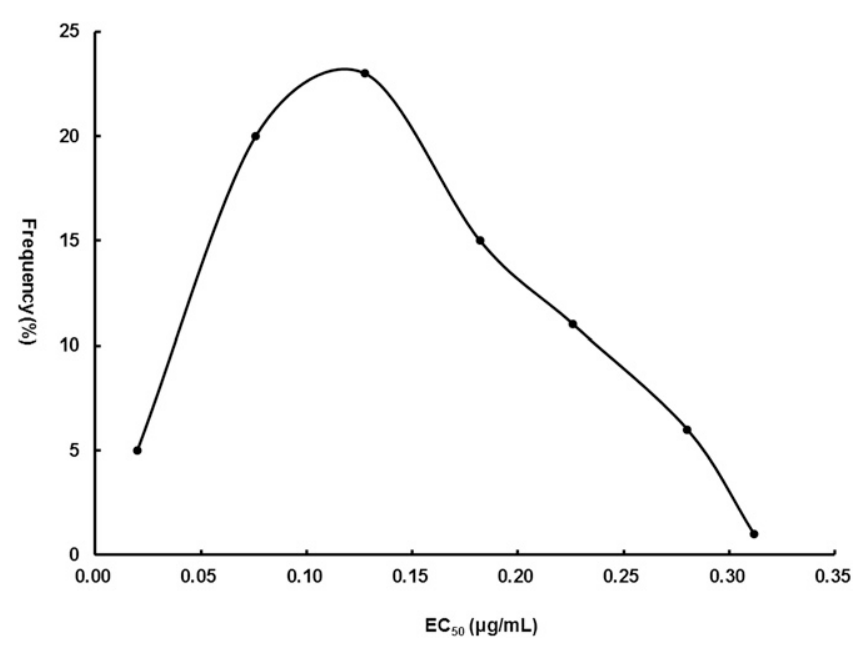

Fig. 6. Frequency distribution of sensitivity of 81 Puccinia strifformis f. sp. tritici isolates to triadimefon. 
suggested that in Petri dishes, delays in symptoms were probably due to the adaptation of leaf segments to the senescence retardant medium (Turra et al. 2017). For statistical analysis of the data, this experiment was different from the field trials, which need many samples to use a grading method. A relatively accurate and simple method was used here to calculate the infected area. This method may also be used to count the area of spots under powdery mildew or downy mildew infections.

As with the traditional pot experiment, the detached leaf assay developed in our study can be used to quantify therapeutic effects of fungicides but is incapable of quantifying their preventive effects. Another disadvantage of this method is to prevent the assay from being used directly on rusts. Nevertheless, previous research has showed that the detached leaf assay was efficient in testing leaf rust control by the fungicides azoxystrobin and tebuconazole in preventive, curative, and eradicative treatments (Turra et al. 2017). The detached leaf assay in this study was also efficient in testing fungicides' preventive effect on wheat stripe rust in favorable conditions, because triadimefon is a penetrating chemical that has all the protective, curative, and eradicative actions and can be absorbed by plant leaves (Reis et al. 2016).

Modern fungicides such as triadimefon are mostly biosynthesis inhibitors or cell division inhibitors, which do not often inhibit the germination of spores but inhibit one or several developmental stages after spore germination when the pathogen coexists with the host (Huang et al. 2001). The method established in this study is actually a tissue-screening method that establishes the interaction system of the fungicide-host-pathogen, making up for the blank of the bioassay using the spore germination method. The present study was able to show that a detached leaf assay could accurately quantify triadimefon sensitivity of Pst within approximately one-eighth of that shown in the traditional pot-grown wheat seedlings experiment, and material costs for it were also much lower than those using pot tests. It was suggested that the method for measuring fungicide effectiveness in an in vitro leaf assay could be accurate, fast, and easy to be controlled. In addition, this method has been used for fungicide selection experiments in our laboratory and field trials (data not shown). The results showed that this novel method could effectively distinguish the efficacy of different fungicides that were verified in field trials. Previous studies showed that it was feasible to study the relevant characteristics of a rust fungus by using the detached leaf bioassay method (Felsenstein et al. 1998; Jackson et al. 2008). A detached leaf bioassay was also developed under controlled laboratory conditions to evaluate the efficacy of five registered fungicides for the control of brown ( $P$. melanocephala) and orange (P. kuehnii) rust of sugarcane, and positive results were also obtained (Chaulagain et al. 2019).

The baseline of triadimefon to Pst was shown to be $0.1453 \mu \mathrm{g} / \mathrm{ml}$, indicating that triadimefon could effectively control wheat stripe rust under the controlled conditions. This was consistent with the results that inhibiting demethylation was fungistatic to rust urediniospores (Mueller et al. 2005). Studies were also conducted to show the efficacy of triazoles to different rust pathosystems under controlled conditions. The pustule development of daylily rust was reduced significantly under greenhouse conditions (Buck and WilliamsWoodward 2003), and quinone outside inhibitor and carboxamide fungicides were proven in vitro very effective for sugarcane rust control (Chaulagain et al. 2019).

In summary, the detached leaf method was proven an excellent technique in the present study, and it can be used to measure the sensitivity and monitor the resistance of the wheat stripe rust fungus to fungicides. With the developments in related subjects such as plant pathology, molecular biology, computer science, instrumental analysis technology, and people's attention to bioassays of fungicides, it is possible to continuously innovate and optimize biometric technologies and to establish more rapid and accurate bioassay methods for quantifying the effectiveness of fungicides on controlling different crop diseases.

\section{Literature Cited}

Bai, Y. R., Mo, X., and Jiang, Y. 2018. Fresh-maintaining effects of different concentrations of 6-BA and B9 on carnation cut flower. Agric. Biotechnol. 7:39-43.

Belan, L. L., Junior, W. C. D. J., Souza, A. F. D., Zambolim, L., Tomaz, M. A., Alves, F. R., and Ferrão, M. A. G. 2015. Monitoring of leaf rust in conilon coffee clones to improve fungicide use. Aus. Plant Pathol. 44:5-12.

Boydom, A., Dawit, W., and Getaneh W/Ab. 2013. Evaluation of detached leaf assay for assessing leaf rust (Puccinia triticina Eriks.) resistance in wheat. J. Plant Pathol. Microbiol. 4:176.

Buck, J. W., and Williams-Woodward, J. L. 2003. The effect of fungicides on urediniospore germination and disease development of daylily rust. Crop Prot. 22:135-140.

Chaulagain, B., Dufault, N., Raid, R. N., and Rott, P. 2019. Sensitivity of two sugarcane rust fungi to fungicides in urediniospore germination and detached leaf bioassays. Crop Prot. 117:86-93.

Chen, W., Wellings, C., Chen, X., Kang, Z., and Liu, T. 2014. Wheat stripe (yellow) rust caused by Puccinia striiformis f. sp. tritici. Mol. Plant Pathol. 15:433-446.

Chen, X. M. 2014. Integration of cultivar resistance and fungicide application for control of wheat stripe rust. Can. J. Plant Pathol. 36:311-326.

CSIC-NY. 2006a. Pesticides guidelines for laboratory bioactivity tests. Part 2: Petri plate test for determining fungicide inhibition of mycelial growth. Page 6 in CSIC-NY NY/T 1156.2-2006. Institute for the Control of Agrochemicals, China

CSIC-NY. 2006b. Pesticides guidelines for laboratory bioactivity tests. Part 4: Potted plant test for fungicide control of powdery mildew on wheat. Page 6 in: CSIC-NY NY/T 1156.4-2006. Institute for the Control of Agrochemicals, China.

CSIC-NY. 2008. Guideline for laboratory bioassay of pesticides. Part 15: Potted plant test for fungicide control leaf rust on cereals. Page 6 in: CSIC-NY NY/ $\mathrm{T}$ 1156.15-2008. Institute for the Control of Agrochemicals, China.

Felsenstein, F. G., Park, R. F., and Zeller, F. J. 1998. The use of detached seedling leaves of Triticum aestivum to study pathogenicity in Puccinia recondita f.sp. tritici. J. Phytopathol. 146:115-121.

Grimmer, M. K., Van, B. F., Powers, S. J., and Paveley, N. D. 2014. Evaluation of a matrix to calculate fungicide resistance risk. Pest Manag. Sci. 70:1008-1016.

He, C. Y., Zhang, Y. H., Zhou, W., Guo, Q. Y., Bai, B., Shen, S. B., and Huang, G. B. 2019. Study on stripe rust (Puccinia striiformis) effect on grain filling and seed morphology building of special winter wheat germplasm Huixianhong. PLoS One 14:e0215066.

Huang, L., Kang, Z., Yan, Y., and Zhang, G. 2001. Effects of ergosterol biosynthesis-inhibiting fungicide triadimefon on the development of Venturia inaequalis on apple leaves. Mycosystema 20:250-257.

Jackson, E. W., Obert, D. E., Chong, J., Avant, J. B., and Bonman, J. M. 2008. Detached-leaf method for propagating Puccinia coronata and assessing crown rust resistance in oat. Plant Dis. 92:1400-1406.

Khandelwal, A., Gupta, S., Gajbhiye, V. T., and Varghese, E. 2014. Degradation of kresoxim-methyl in soil: Impact of varying moisture, organic matter, soil sterilization, soil type, light and atmospheric $\mathrm{CO}_{2}$ level. Chemosphere 111:209-217.

Lin, C. Y. 1982. Preliminary research on triadimefon as a seed dressing for controlling epidemics of yellow rust of wheat. J. Plant Prot. 4:265-270.

Liu, X. 2004. Baseline Sensitivity of Pseudoperonospora cubensis and Alternaria solani to Azoxystrobin and Its Resistance Risk Assessment. Nanjing Agricultural College, Nanjing, China.

Mueller, D. S., Jeffers, S. N., and Buck, J. W. 2005. Toxicity of fungicides to urediniospores of six rust fungi that occur on ornamental crops. Plant Dis. 89:255-261.

Patial, M., Kumar, J., and Pal, D. 2017. Detached leaf assay for evaluating resistance to leaf rust Pst. 104-2 in wheat (Triticum aestivum L.). Indian J. Exp. Biol. 55:789-794.

Pfaller, M. A., Sheehan, D. J., and Rex, J. H. 2004. Determination of fungicidal activities against yeasts and molds: Lessons learned from bactericidal testing and the need for standardization. Clin. Microbiol. Rev. 17:268-280.

Reis, E. M., Zanatta, T., and Zanatta, M. 2016. Curative and eradicant action of fungicides to control Phakopsora pachyrhizi in soybean plants. Summa Phytopathol. 42:295-302.

Sergiev, I., Todorova, D., Somleva, M., Alexieva, V., Karanov, E., Stanoeva, E., Lachkova, V., Smith, A., and Hall, M. 2007. Influence of cytokinins and novel cytokinin antagonists on the senescence of detached leaves of Arabidopsis thaliana. Biol. Plant. 51:377-380.

Shang, H., and Lu, H. 1991. Therapeutic effect of triadimefon on wheat stripe rust. J. Northwest Agric. Univ. S1:39-43.

Siegel, M. R. 1981. Sterol-inhibiting fungicides: Effects on sterol biosynthesis and sites of action. Plant Dis. 65:986-989.

Turra, C., Reis, E. M., and Barcellos, A. L. 2017. Detached wheat leaf assay for assessing the sensitivity of Puccinia triticina races to fungicides. Summa Phytopathol. 43:181-185.

Wang, F. F., Wang, Z., Zhang, B. H., and Zhang, Q. M. 2017. Degradation and adsorption of tebuconazole and tribenuron-methyl in wheat soil, alone and in combination. Chil. J. Agric. Res. 77:281-286.

Wellings, C. R. 2011. Global status of stripe rust: A review of historical and current threats. Euphytica 179:129-141.

Yang, Y., Chen, Y., Chen, F., Yu, Y., and Bi, C. 2017. Tea polyphenol is a potential antifungal agent for the control of obligate biotrophic fungus in plants. J. Phytopathol. 165:547-553. 